\title{
Diagnostic Accuracy of Cytological Sampling Techniques by Bronchoscopy in the Diagnosis of Lung Cancer
}

\author{
Saumya Agrawal ${ }^{1}$ and Mamta Gupta ${ }^{2 *}$ \\ 'Department of ENT, Dr B.R. Ambedkar Medical College, Bangalore, India \\ ${ }^{2}$ Department of Pathology, Subharti Medical College, Meerut. India
}

\begin{abstract}
Background: With advent of newer diagnostic modalities for obtaining small biopsies and cytologic samples by bronchoscopy it is important to assess the diagnostic accuracy of these techniques.

Material and Methods: A Retrospective and Prospective study was conducted on 347 bronchoscopic specimens from 200 patients with clinical and / or radiological suspicion of carcinoma lung. To evaluate the role and relative diagnostic accuracies of bronchial washings, brushings and broncho-alveolar lavage in the diagnosis of lung cancer

Results: $119 / 200$ cases were diagnosed as positive for malignancy on cytology and 56/80 cases were confirmed positive on histopathological evaluation. Cytological materials included in the study were $8 \mathrm{BW}, 180 \mathrm{BAL}$ and $159 \mathrm{BB}$ constituting a total sample size of 347 . The overall sensitivity of BW, BAL and BB was $25 \%, 72 \%$ and $94 \%$ respectively. Thus, BB provided a significantly superior sensitivity and improved the diagnostic yield. Squamous cell carcinoma was the most common histologic type of malignancy diagnosed on both cytology and histopathologic evaluation with accuracy of $78.8 \%$. A specific diagnosis of malignancy was made in 124 cases (62\%) by combination of cytology and biopsy.
\end{abstract}

Conclusion: Cytologic samples and small biopsies obtained under bronchoscopic guidance are forming important samples for diagnosing and classifying lung tumours. Every technique has utility and limitation. However, combination of different methodologies on bronchoscopy increases the diagnostic accuracy.

Keywords: Bronchoscopy, Bronchial Brushing, Broncho-Alveolar Lavage, Bronchial Biopsy, Cytology, Histopathology

\section{Introduction}

Lung cancer continues to be the leading cause of cancer deaths. Despite the great progress made in the treatment of other cancers in recent decades, the 5-year survival of patients with lung cancer has remained poor, ranging from $6 \%$ to $14 \%$ for men and $7 \%$ to $18 \%$ for women. ${ }^{[1]}$

It is usually suspected on the basis of an abnormal radiographic imaging study, in conjunction with symptoms caused by local or systemic effects of the tumor. ${ }^{[2]}$ The diagnosis of lung cancer by cytologic methods is of historic interest because it was an early demonstration that malignancy could be diagnosed by examining exfoliated cells. ${ }^{[3]}$

There have been significant advances in the early diagnosis of lung cancer in high risk patient groups using bronchoscopic methods. ${ }^{[4]}$ When the results of sputum studies are negative and clinical suspicion persists, or when lesions are located in the pulmonary periphery, fiberoptic bronchoscopy may be used to obtain diagnostic cellular material. ${ }^{[5]}$

The use of lung cytology has evolved in the last few years. Emphasis has shifted from diagnosis of malignancy in inoperable patients and confirmation of metastatic tumors to its use as a definitive diagnostic procedure on which crucial patient management decisions are based. ${ }^{[6]}$

Flexible fiber-optic bronchoscope has revolutionized the respiratory cytology, as techniques like bronchial washings, bronchial brushings, broncho-alveolar lavage, trans-bronchial needle aspirations and bronchial biopsy have become more easy, accessible and popular. ${ }^{[7,8]}$ Also the recent classification highlights that small biopsies and cytology specimens play an important role in diagnosis and should be handled carefully. ${ }^{[9]}$

Therefore, this study is undertaken to evaluate the role and diagnostic accuracy of bronchial washings, bronchoalveolar lavage, bronchial brushings and bronchial biopsy in the diagnosis of lung cancer.

\section{Materials and Methods}

Retrospective and Prospective study was conducted at Department of Pathology, Kasturba Medical College, Mangalore over a period of four years. 347 bronchoscopic specimens from 200 patients with clinical and / or radiological suspicion of lung carcinoma were collected. 
Cases in which the sample was inadequate on cytologic examination were excluded. Ethical clearance was taken prior to study.

Procedure: For retrospective analysis, data from pathology files was taken to identify all cases for which cytology and biopsy specimens were obtained by bronchoscopic procedure. Clinical and radiologic data was taken from medical files.

For prospective study, clinical and radiological findings were obtained from the files and cytological samples obtained by flexible fiber-optic bronchoscopy done by the pulmonologist were sent to the laboratory for processing.

(a) Bronchial brushings were received as air dried and wet-fixed smears of two to three brushings by disposable bronchial brush, smeared directly on to clean glass slides. The air dried smears were stained with May-Grunwald Geimsa and the wet fixed slide with Papanicolaou stain.

(b) Bronchial washing and Broncho-alveolar lavage samples were received as $20 \mathrm{ml}$ aliquots of normal saline in sterile vials. Samples were centrifuged at $3000 \mathrm{rpm}$ for 5 minutes and prepared into air-dried and wet-fixed smears. There were 4 smears in total, 2 smears for pap-stain, 1 smear for hematoxylin and eosin (H\&E)staining and 1 smear for AFB staining.

(c) Bronchial biopsies were received in $10 \%$ formalin and processed routinely to obtain 4-5 $\mu$ thick paraffin sections and stained with $\mathrm{H} \& \mathrm{E}$ stain.

All these cases were categorized into four groups based on the cytological features as-

Group A- positive for malignancy

Group B- suspicious for malignancy

Group C- Atypical features

Group D- Negative for malignancy

Diagnostic accuracy of the various cytological sampling techniques was evaluated by comparing with the cytological diagnosis. Correlation with bronchial biopsy was done wherever available. Biopsy specimens showing dysplasia, carcinoma in situ, invasive carcinoma, or other malignancies were considered positive. The neoplastic lesions were typed based on the WHO classification of lung tumors.

Data Analysis: The collected data was entered in and analysed using Statistical Package for Social Sciences (SPSS) version 11.5 and association was found by using chi square test.

\section{Results}

347 bronchoscopic specimens were obtained from 200 patients with a clinical diagnosis of bronchogenic carcinoma during the study period. The cytologic material included brochoalveolar lavage constituting $51.8 \%$, bronchial washings $2.3 \%$ and bronchial brushings $45.8 \%$ of the total number of samples received. Both bronchoalveolar lavage and bronchial brushings were obtained from 146 patients, bronchial washings and brushings from one patient and only bronchoalveolar lavage, bronchial washings and brushings from 34, 7 and 12 patients respectively. (Figure 1)

The patients were in the age range of 27 to 87 years with majority of patients presenting in the 6th decade of life. A clinical diagnosis of lung carcinoma was made in 162 males $(81 \%)$ while females constituted only $38(19 \%)$ of cases. The commonest symptom with which the patients presented was cough in $60 \%$ of cases followed by breathlessness $(43 \%)$, hemoptysis $(25 \%)$ chest pain $(18 \%)$ and weight loss (40\%).

Bronchoscopic Findings: Exophytic growth /nodules (39\%) were the most common observed findings in the patients followed by mucosal abnormality/ inflammation $(32 \%)$ and Endophytic or infiltrative growth (11.5\%).

Cytological Diagnosis: Bronchoalveolar lavage was collected from 180 patients of which a diagnosis of malignancy was made in $41.1 \%$ of the cases. Bronchial brushings were performed in 159 cases. All cases had mucosal irregularity or growth on bronchoscopic examination. A diagnosis of malignancy was made in $61.7 \%$ of cases. Bronchial washings were done in 8 cases of which 2 cases were positive (Table 1)

The diagnostic accuracy of detection of malignant cells in various samples was analyzed. For calculation of diagnostic correlation between different cytologic samples, Suspicious category was included in positive for malignancy and atypical cases were considered negative for malignancy.

Both bronchoalveolar lavage and bronchial brushings were collected from 146 patients. 52 cases showed no malignancy. One case which was non-malignant on bronchial brushings was diagnosed as adenocarcinoma on bronchoalveolar lavage and was confirmed on biopsy. This lesion was located in the peripheral part of lung in left lower lobe on CT scan. 20 cases were positive for malignancy on brush smears for which the bronchoalveolar lavage was negative, of these eleven cases were diagnosed as squamous cell carcinoma, five were adenocarcinoma, 2 small cell carcinomas, 1 poorly differentiated carcinoma and one was suspicious for malignancy (Figure 2). Diagnostic 
accuracy for detection of malignancy by bronchoalveolar lavage in correlation with bronchial brushings was $85.6 \%$, Sensitivity was $78 \%$, Specificity $98 \%$, Positive predictive value $99 \%$ and Negative predictive value $72 \%$. Correlation between findings of BAL and Bronchial brushings were significant. ( $p$ value $<0.0001$ ) Both bronchial washing and bronchial brushing were obtained from only one case. It was positive on both with diagnostic concordance of $100 \%$.

Cytological diagnosis of malignancy was made in 119/200 cases $(59.5 \%)$ in which a clinical suspicion of carcinoma lung was made. Squamous cell carcinoma was the most common diagnosed malignancy in 58 cases followed by adenocarcinoma in 32 cases. (Table 2)

Bronchial biopsy was obtained from $81 / 200$ cases $(40.5 \%)$ that underwent bronchoscopy. Biopsy specimens showing dysplasia, carcinoma in situ, invasive carcinoma, or other malignancies were considered positive. One biopsy was inadequate for opinion, however on brushings it was diagnosed as squamous cell carcinoma. On biopsy also squamous cell carcinoma was the most common diagnosed malignancy in $46.3 \%$ of cases. (Table 3 )

Two cases in which bronchial washings were collected, also had a biopsy correlation. One case was squamous cell carcinoma and other was adenocarcinoma. The diagnostic accuracy for malignancy was $100 \%$ in both these cases.

Bronchoalveolar lavage and bronchial biopsy was done in 75 patients. Diagnostic accuracy of bronchoalveolar lavage in correlation with bronchial biopsy was $63.8 \%$, Sensitivity $72 \%$, specificity $60 \%$, Positive predictive value $78 \%$, Negative predictive value $52 \%$ and $p$ value was significant $(\chi 2=0.007)$
Bronchial brushings and biopsy was done in 70 patients. Diagnostic accuracy of bronchial brushings in correlation with bronchial biopsy was $80 \%$, Sensitivity was $94 \%$, Specificity $48 \%$, Positive predictive value $81 \%$, Negative predictive value $77 \%$, p value was Significant $(\chi 2<0.0001)$.

Thus, overall correlation between final cytological diagnosis and histopathological diagnosis showed a diagnostic accuracy of a malignant diagnosis in $77.5 \%$ of cases. Sensitivity was $91 \%$, Specificity $46 \%$, Positive predictive value $80 \%$ and Negative predictive value $69 \%$.

The diagnostic accuracy of interpretation of SCC was $78.8 \%$ (Sensitivity $76 \%$, Specificity $81 \%$, PPV 78\%, NPV $80 \%$ ). One case was interpreted as positive for dysplasia on biopsy however cytological diagnosis was SCC. 2 cases were misinterpreted as adenocarcinoma on cytology. Adenocarcinoma was second most common histologic type with a diagnostic accuracy of $88.7 \%$ (Sensitivity $82 \%$, Specificity 90\% PPV 56\%, NPV 97\%). Small cell carcinoma was diagnosed in 3 cases on cytology for which biopsy correlation was available, however one was negative on biopsy. Of the 4 cases diagnosed as non-small cell carcinoma, 2 cases were moderately differentiated squamous cell carcinoma, one case was large cell carcinoma and one was negative for malignancy. 2 cases suspicious on cytology were negative on biopsy. 5 cases were diagnosed negative on cytology, but were proved to be malignant on biopsy. Thus, a specific diagnosis of malignancy was made in 124 cases $(62 \%)$ by combination of cytology and biopsy.

Table 1: Cytomorphological diagnosis on different cytological samples.

\begin{tabular}{|c|c|c|c|c|c|c|c|c|}
\hline \multirow{3}{*}{ Cytological Diagnosis } & \multicolumn{6}{|c|}{ Cytological Samples } & \multicolumn{2}{|c|}{ Total Patients } \\
\hline & \multicolumn{2}{|c|}{ Bronchalveolar lavage } & \multicolumn{2}{|c|}{ Bronchial brushings } & \multicolumn{2}{|c|}{ Bronchial washings } & \multirow[b]{2}{*}{ Number } & \multirow[b]{2}{*}{$\%$} \\
\hline & Number & $\%$ & Number & $\%$ & Number & $\%$ & & \\
\hline Positive for malignancy & 74 & 41.1 & 98 & 61.7 & 2 & 25 & 119 & 59.5 \\
\hline Squamous cell carcinoma & 41 & & 52 & & 1 & & 58 & 29 \\
\hline Adenocarcinoma & 23 & & 30 & & 1 & & 32 & 16 \\
\hline Small cell carcinoma & 3 & & 5 & & 0 & & 5 & 2.5 \\
\hline Adenosquamous carcinoma & 0 & & 1 & & 0 & & 1 & 0.5 \\
\hline Poorly differentiated & 3 & & 5 & & 0 & & 4 & 2.0 \\
\hline Non- small cell carcinoma & 4 & & 5 & & 0 & & 5 & 2.5 \\
\hline
\end{tabular}




\begin{tabular}{|c|c|c|c|c|c|c|c|c|}
\hline Suspicious & 9 & 5.0 & 5 & 3.1 & 0 & 0 & 6 & 3.0 \\
\hline Atypical & 10 & 5.5 & 5 & 3.1 & 0 & 0 & 8 & 4.0 \\
\hline Negative & 87 & 48.4 & 51 & 32.1 & 6 & 75 & 81 & 40.5 \\
\hline Total (347) & 180 & & 159 & & 8 & & 200 & 100 \\
\hline
\end{tabular}

Table 2 : Histopathological diagnosis on bronchial biopsy

\begin{tabular}{|l|c|c|}
\hline Histopathological Diagnosis & No. of patients & Percentage \\
\hline Squamous cell carcinoma & 37 & 46.3 \\
\hline Adenocarcinoma & 12 & 15 \\
\hline Small cell carcinoma & 2 & 2.5 \\
\hline Poorly differentiated carcinoma & 3 & 3.8 \\
\hline Large cell carcinoma & 1 & 1.2 \\
\hline Dysplasia & 1 & 1.2 \\
\hline Negative & 24 & 30 \\
\hline Total & $\mathbf{8 0}$ & 100 \\
\hline
\end{tabular}

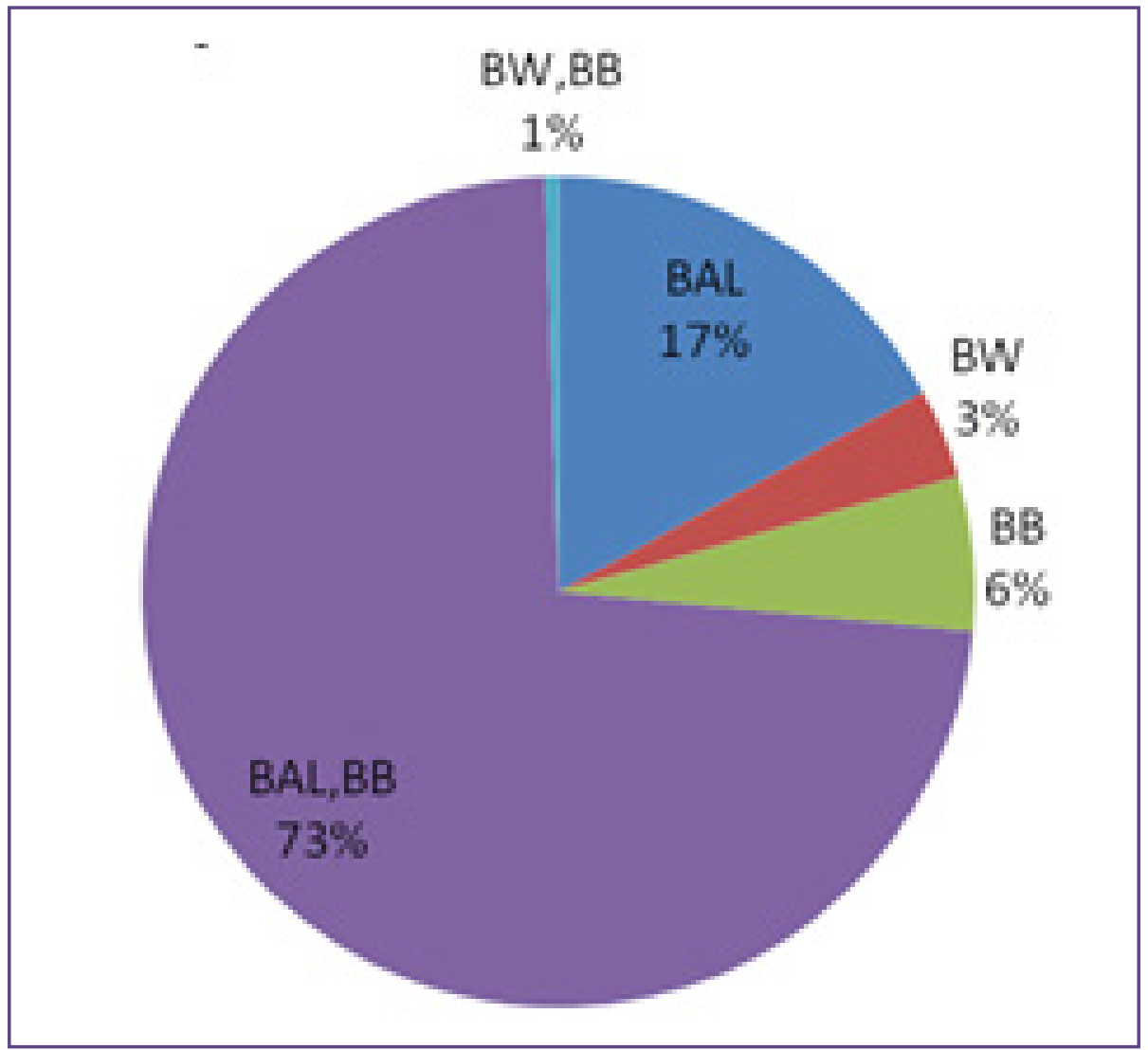

Fig. 1: Nature of cytology samples. 


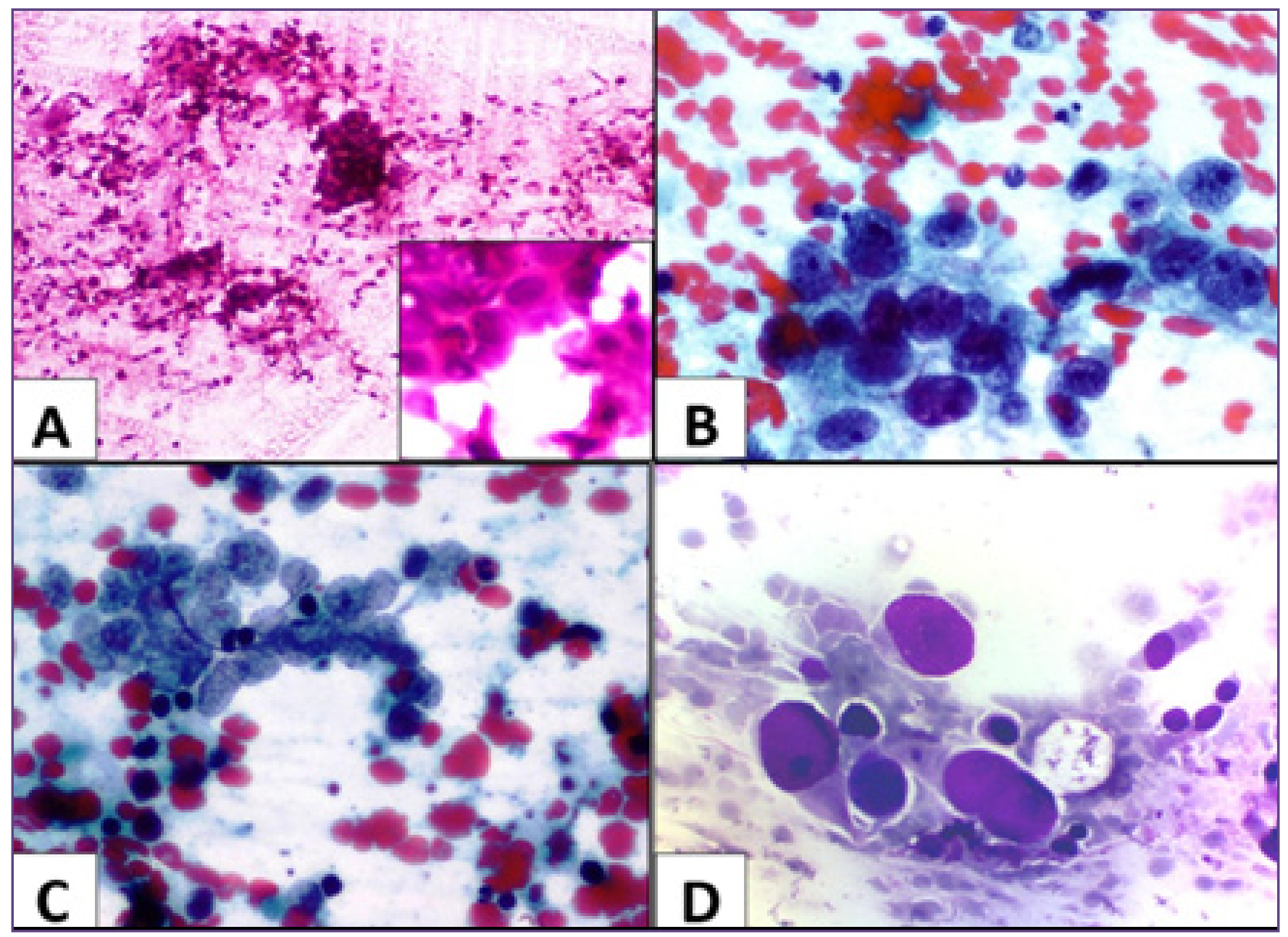

Fig. 2: A. BAL fluid- Squamous cell carcinoma , H\&E,40X (Inset 400X) B. Bronchial brush -Adenocarcinoma, Pap, 400X C. Bronchial brush - Small cell carcinoma, Pap, 400X D. Bronchial brush - Poorly differentiated carcinoma, MGG, 400X.

\section{Discussion}

Fibreoptic bronchoscopy has been routinely used for the diagnosis of suspected lung cancer. ${ }^{[10]}$ The reported diagnostic yield from bronchoscopies in patients with lung cancer varies greatly. The optimal combination of sampling techniques has not been finally established. ${ }^{[11]}$

In this study we evaluated the diagnostic accuracies of bronchial washings, bronchoalveolar lavage and bronchial brushings obtained during bronchoscopic examination. We observed that a combined use of these techniques increase the diagnostic rate by $86.5 \%$. Similar observation was also observed by Kawaraya M et al. ${ }^{[10]}(93.5 \%)$ and Chaudhary BA et al. ${ }^{[12]}(95.8 \%)$.

Most of the patients were in the $6^{\text {th }}$ decade of life (mean 64 years). Similar observations have been observed by Sackett MK et a ${ }^{[7]}$ and Sturgis $C D$ et a ${ }^{[5]}$ where mean age of presentation was 67 and 65 years respectively.
The incidence in men has been falling steadily since the 1970 s, but is rising in women. These changes reflect smoking behaviour. Survival rates with lung cancer are very poor, so mortality has a similar pattern to incidence. Lung cancer causes $26 \%$ of male and $17 \%$ of female cancer deaths. ${ }^{[13]}$ In the present study, majority ( $81 \%$ ) cases were males.

Bronchoscopy is a useful procedure for both endoscopically visible and non- visible lesions as in case of peripheral lesions. ${ }^{[14]}$ In the present study, $17.5 \%$ cases showed no lesions, while mucosal irregularity or inflammation, obvious growth and infiltrative lesions were seen in $32 \%$, $39 \%$ and $11.5 \%$ of cases respectively.

The choice of cell collection technique is influenced by factors such as the personal preference of the physician, the status of the patient, the location of the lesion, and the differential diagnosis. However, the cytologist should understand that while cells obtained by different techniques 
have substantially similar morphologic characteristics, important differences are caused by differences in cell preservation and specimen processing. ${ }^{[3]}$

There remains a debate as to whether a cytologic diagnosis of lung cancer needs to be confirmed by biopsy. According to various authors, diagnostic utility of bronchial washings and brushings vary among different types of lung carcinoma, so a biopsy is required, however others have suggested that cytology should be considered for definite diagnosis and classification of lung cancers without the need for histopathologic confirmation. ${ }^{[15]}$

Cytological sampling by BAL technique relies mainly on cells 'exfoliated' from the malignant lesion in the bronchial epithelium. In general, less differentiated, anaplastic lesions have more loosely cohesive cells and exfoliate more in comparison to well differentiated lesions. Secondly, while these exfoliated cells are lying in the bronchus, they may develop degenerative changes, and lose their morphological details which are important in differentiating them from non-malignant cells shed off by the normal bronchial epithelial lining. If the technique of the pulmonologist is not proper, lesser cytological material is retrieved, increasing the chances of false negative results. ${ }^{[8]}$

Bronchoalveolar lavage is a useful diagnostic tool in diffuse or disseminated lung malignancies that do not involve the bronchial structures visible by endoscopy. The neoplastic histological type and the intraparenchymal neoplastic growth pattern are good predictors for diagnostic yield; adenocarcinoma, and tumors with lymphangitic or lepidic growth patterns are more easily diagnosed by bronchoalveolar lavage; in these cases the diagnostic yield reported is higher than $80 \%{ }^{[16]}$

In the present study, 180 samples of BAL were collected, a diagnosis of malignancy was made in $41.1 \%$ of cases, Poletti $\mathrm{V}$ et $\mathrm{a}^{[17]}$ in a study of 162 cases of malignant cell infiltrates in the lung diagnosed malignancy in $76 \%$ of the cases. The diagnostic accuracy varied depending on the neoplastic nature and growth pattern of the disease. Gaur DS et $\mathrm{al}^{[8]}$ in a study on efficacy of BAL, observed that the sensitivity, specificity and accuracy of BAL samples $39.4 \%, 89.6 \%$ and $71.4 \%$ respectively, which were similar to our study.

Sigari $\mathrm{N}$ et $\mathrm{al}^{[18]}$ in a study to compare the diagnostic value of bronchial washing in lung cancer before and after biopsy, observed that the sensitivity of cytology of bronchial washing was 31.25 percent. They concluded that bronchial washing cytology is not recommended as a routine procedure for diagnosis of lung cancer but if biopsy is contraindicated this procedure may be helpful. In our study, BW constituted $2.2 \%$ of the total samples and malignancy was diagnosed in $2(25 \%)$ of cases. Flint A et al ${ }^{[19]}$ observed that diagnostic yield of BW may be increased if cell blocks are prepared and analyzed.

Although the complimentary role of various conventional cytological methods is well recognized, bronchial brushing is the only single technique that significantly improved the diagnostic yield. In the present study a malignant diagnosis was made in $61.7 \%$, which was more when compared to lavage and washings.

Several studies have compared the diagnostic accuracies of cytology and histopathology obtained during the bronchoscopic procedure. In the present study, washings and brushings ( 8 cases) when compared had a sensitivity of $25 \%$. Biopsy was not available in any of these cases, but since the number of samples was less for comparison, the exact accuracy could not be determined. Mak VH ${ }^{[20]}$ in a study on value of washings and brushings at fibrooptic bronchoscopy concluded that if the lesion is visible, the accuracy is $95 \%$ with either technique. However, if the tumor is not visible on endoscopy a combination of biopsy and washings was more successful (94.3\%) than biopsy and brushing $(82.8 \%)$.

In the present study, the sensitivity of BAL and brushing was $72 \%$ and $94 \%$ respectively. Thus $\mathrm{BB}$ provided a significantly superior sensitivity of $94 \%$. Various workers have tried to combine the two techniques of $\mathrm{BB}$ and $\mathrm{BAL}$ to improve the yield of diagnostic cytological material. Studies by other authors showed similar sensitivity patterns. Govert JA et al ${ }^{[14]}$ and Chopra SK et $\mathrm{al}^{[21]}$ reported a sensitivity of $85.3 \%$ and $86.3 \%$ which were comparable to our study.

Saita $\mathrm{S}$ et al ${ }^{[22]}$ in a comparative evaluation of BB and biopsy in diagnosing visible bronchial lesions concluded that brushings showed a higher sensitivity $(85.6 \%)$ than biopsy. A combination of two techniques gave an accuracy rate of $94.3 \%$. In the present study, sensitivity of BAL in correlation with biopsy and brushings with biopsy was $72 \%$ and 94\% respectively, achieving a diagnostic accuracy for malignancy in $77.5 \%$ of the cases.

Comparing cytological and histopathological type of malignancy, diagnostic accuracy of interpretation of squamous cell carcinoma was $78.8 \%$ followed by adenocarcinoma with accuracy of $88.7 \%$. Small cell carcinoma was diagnosed in $3.5 \%$ of the cases.

In a large study by Johnston and Frable, the prediction of histologic type of primary lung cancer from bronchial material was compared with histological diagnosis. 
The rate of concordance was $85 \%$ with SCC, $79 \%$ with adenocarcinoma, and $93 \%$ with small cell carcinoma. The variable sampling of different areas within a tumor can result in poor correlation between cytological and histological diagnosis of small cell carcinoma as this is important in selection of therapy. ${ }^{[3]}$

Similar observations were observed by Matsuba $\mathrm{M}^{[23]}$ in a study comparing diagnostic accuracy of BB with biopsy and cell typing reliability observed $90.3 \%$ and $64.8 \%$ positive results on brushings and biopsy respectively. The combination of both the techniques yielded a positive diagnosis in $93.7 \%$ of cases. In their study, there was a high incidence of positive diagnosis for SCC and small cell carcinoma, as was in the present study. The cell typing accuracy of two methods is similar and the results obtained by both the techniques may be taken into consideration in the management of individual cases of lung cancer. ${ }^{[23]}$

Rarely discordance may result by interpreting sub-optimal specimens, misinterpreting squamous dysplasia as SCC on the cytology specimen, misinterpreting a poorly differentiated neoplasm and not recognizing a second component of a combined SCLC-NSCLC. ${ }^{[7]}$ In our study, one case was misinterpreted as SCC, which on histology gave squamous dysplasia. And on biopsy, two cases of PDC were reclassified as SCC. Two cases of NSCLC were diagnosed as squamous cell carcinoma. Thus, challenging cases and unusual diagnosis and sub-optimal specimens should prompt reconciliation of findings between cytology and biopsy specimens, as well as with pertinent previous histologic specimens before finalizing the diagnosis. ${ }^{[7]}$

In the present study, the difficulties encountered in cytological evaluation could be attributed to the location of lesion, nature of material obtained, processing technique and difficulty in typing of tumour. Tumours easily identifiable on flexible bronchoscopy, central location gave better yield with most of the techniques. However, peripherally situated lesions were better sampled by BAL. However, sample degeneration occurred if processing time was delayed in BAL and BW creating difficulty in reporting. Brushings showed best preservation of morphological details and highest diagnostic accuracy. Rarely, biopsies were negative for tumour while cytology was positive, this could be due to sampling error. The sequence of sampling should be lavage or washings followed by brushings and biopsy so that blood obtained in the latter technique do not obscure morphological details. Sometimes, it is not possible to definitely classify a lesion on cytology. This especially occurs with poorly differentiated tumours, non-small cell carcinoma and reactive conditions mimicking neoplasia. In all cases clinico-radiological correlation is important.

\section{Conclusion}

The role of any diagnostic technique refers to its utility and limitations. However, diagnostic accuracy may be enhanced by combining various techniques. Cytology of bronchial washings, bronchoalveolar lavage and bronchial brushings are highly sensitive. Proper collection of the sample, adequate fixation, prompt processing and staining are essential. In present times, the classification of lung carcinomas are largely being based on cytologic specimens and is becoming one of the most challenging areas of diagnostic cytopathology. Morphologic examination alone is sufficient in cases of well and moderately differentiated carcinoma. However, the accurate classification of NSCLC is often challenging and not reproducible with poorly differentiated carcinomas. ${ }^{[24]}$ With the development of targeted therapies of which the efficacy and toxic effects are linked to histologic type of lung carcinoma, different diagnostic approach to lung carcinomas on small specimens needs to be considered and warrants further studies in future.

Acknowledgement and funding - ICMR, New Delhi for accepting this manuscript for STS project 2011

\section{References}

1. Cagle PT, Allen TC, Dacic S, Beasley MB, Borczuk AC, Chirieac LR, Laucirica R, et al. Revolution in Lung Cancer New Challenges for the Surgical Pathologist. Arch Pathol Lab Med. 2011;135:110-16.

2. Gilbert Schreiber G, McCrory DC. Performance Characteristics of different modalities for diagnosis of suspected lung cancer. Chest 2003; 123:115S-128S.

3. Linder J. Lung Cancer Cytology. Something Old, Something NewAm J ClinPathol 2000;114:169-171.

4. Kamath AV, Chhajed PN Role of Bronchoscopy in Early Diagnosis of Lung Cancer. Indian J Chest Dis Allied Sci 2006; 48: 265-9.

5. Sturgis CD, Nassar DL, D'Antonio JA, Raab SS. Cytologic features useful for distinguishing small cell from non-small cell carcinoma in bronchial brush and wash specimens. Am J ClinPathol 2000;114:197-202.

6. Bhatia A, Singh N, Arora VK.A Perspective on Cytology of Lung Cancer. Indian J Chest Dis Allied Sci 2004; 46: 81-3.

7. Sackett MK, Salomao DR, Donovan JL, Yi ES, Aubry MC, Diagnostic Concordance of Histologic Lung Cancer Type between Bronchial Biopsy and Cytology Specimens taken during the Same Bronchoscopic Procedure. Arch Pathol Lab Med. 2010;134:1504-12.

8. Gaur DS, Thapliyal NC, Kishore S, Pathak VP. Efficacy of Broncho-Alveolar Lavage and Bronchial brush cytology in diagnosing lung cancers. Journal of Cytology 2007;24(2): 73-77.

9. Travis WD, Brambilla E, Nicholson AG, Yatabe Y, Austin JHM, Beasley MB, et al. The 2015 World Health Organization 
classification of lung tumors impact of genetic, clinical and radiologic advances since the 2004 classification. J Thorac Oncol. 2015;10: 1243-1260.

10. Kawaraya M, Gemba K, Ueoka H, Nishii K, Kiura K, Kodani $\mathrm{T}$, et al.. Evaluation of various cytological examinations by bronchoscopy in the diagnosis of peripheral lung cancer. $\mathrm{Br}$ J Cancer. 2003; 89(10): 1885-8.

11. Roth K, Hardie1 JA, Andreassen AH, Leh F, Eagan TML.Predictors of diagnostic yield in bronchoscopy: a retrospective cohort study comparing different combinations of sampling techniques.BMC Pulmonary Medicine 2008, 8:2 URL http://www.biomedcentral.com/1471-2466/8/2

12. Chaudhary BA, Yoneda K, Burki NK. Fiberoptic bronchoscopy. Comparison of procedures used in the diagnosis of lung cancer. J ThoracCardiovasc Surg. 1978 Jul;76(1):33-7.

13. Hamilton W and Sharp D. Diagnosis of lung cancer in primary care: a structured review. Family Practice 2004; 21: 605-611.

14. Govert JA, Kopita JM, Matchar D, Kussin PS, Samuelson WM. Cost-effectiveness of collecting routine cytologic specimens during fiberoptic bronchoscopy for endoscopically visible lung tumors. Chest. 1996;109:451-6.

15. Truong LD, Underwood RD, Greenberg SD, McLarty JW. Diagnosis and typing of lung carcinomas by cytopathologic methods. Acta Cytol 1985;29(3):379-84.

16. Poletti V, Poletti G, Murer B, Saragoni L, Chilosi M. Bronchoalveolar lavage in malignancy. Semin Respir Crit Care Med. 2007;28(5):534-45.
17. Poletti V, Romagna M, Allen KA, Gasponi A, Spiga L. Bronchoalveolar lavage in the diagnosis of disseminated lung tumors. ActaCytol. 1995;39(3):472-7.

18. Sigari N, Poor Anaraki Z, Salehian P, Safavi A, Ensani F, Accuracy of bronchial washing cytology before and after biopsy for lung cancer diagnosis Scientific Journal of Kurdistan University of Medical Sciences, 2006;10(4):1-5.

19. Flint A. Detection of pulmonary neoplasms by bronchial washings are cell blocks a diagnostic aid? Acta Cytol 1993;37;(1):21-3.

20. Mak VH, Johnston ID, Hetzel MR, Grubb C. Value of washings and brushings at fibreoptic bronchoscopy in the diagnosis of lung cancer. Thorax. 1990 May;45(5):373-6.

21. Chopra SK, Genovesi MG, Simmons DH, Gothe B. Fibreoptic bronchoscopy in the diagnosis of pre and post bronchoscopy sputa, washings, brushings and biopsies. ActaCytol 1977;21(4):524-7.

22. Saita S, Tanzillo A, Riscica C, Maresca A, Potenza E and D'Arrigo M. Bronchial brushing and biopsy: a comparative evaluation in diagnosing visible bronchial lesions.European Journal of Cardio-Thoracic Surgery1990;4:270-272.

23. Matsuda M, Horai T, Nakamura S, Nishio H, Sakuma T, Ikegami $\mathrm{H}$, et al. Bronchial brushing and bronchial biopsy: comparison of diagnostic accuracy and cell typing reliability in lung cancer. Thorax 1986;41:475-8.

24. Ocque R, Tochigi N, Ohori NP, Dacic S Usefulness of Immunohistochemical and histochemical studies in the classification of lung adenocarcinoma and squamous cell carcinoma in cytologic specimens. Am J Clin Pathol 2011;136:81-7.

*Corresponding author:

Dr Mamta Gupta, Associate Professor, Department of Pathology, Subharti Medical College, Meerut 250002 INDIA

Email: drmamta04@gmail.com,drmamtagupta2016@gmail.com

Financial or other Competing Interests: None. 